\title{
Observable Solitonic Features of the Generalized Reaction Duffing Model
}

\author{
Bo Tian ${ }^{\mathrm{a}, \mathrm{b}}$ and Yi-Tian $\mathrm{Gao}^{\mathrm{c}}$ \\ ${ }^{a}$ School of Science, Beijing University of Posts and Telecommunications, Bejing 100876, China \\ ${ }^{\mathrm{b}}$ National Laboratory of Software Development Environment, Beijing University of Aeronautics \\ and Astronautics, Beijing 100083, China \\ ${ }^{\mathrm{c}}$ Institute of Fluid Mechanics, Beijing University of Aeronautics and Astronautics, \\ Beijing 100083, China
}

Reprint requests to the corresponding author, Prof. Y.-T. G.; gaoyt@ public.bta.net.cn

Z. Naturforsch. 57 a, 39-44 (2002); received November 27, 2001

\begin{abstract}
In engineering and physical sciences, solitons and nonlinear evolution equations are of current interest. To the generalized reaction Duffing model, we report several families of exact solitonic solutions, including shock waves and bell-shaped waves. Some of the observable solitonic features are pictured out.
\end{abstract}

Key words: Generalized Reaction Duffing Model; Generalized Hyperbolic-function Method; Observable Solitonic Features; Nonlinear Evolution Equations; Computerized Symbolic Computation.

In many fields of engineering and physical sciences, solitons and nonlinear evolution equations are a major subject. The generalized reaction Duffing equation

$$
u_{t t}+a u_{x x}+b u+c u^{2}+d u^{3}=0
$$

covers such famous ones as the Klein-Gordon, Landau-Ginzburg-Higgs and $\phi^{4}$ equations [1 - 3], where $a, b, c$ and $d$ are all constants. Several solitary-wave and periodic solutions of (1) have recently been reported in [4]. Topics on the Duffing equation in mechanical engineering can be seen, e.g., in [5] and references therein.

In this paper, we investigate (1) for its solitonic features by considering the general case $a \neq 0, d \neq 0$.

By virtue of the generalized hyperbolic-function method [6] we assume that the exact solutions of (1) be

$$
\begin{array}{r}
u(x, t)=\sum_{m=0}^{M} E_{m}(t) \cdot \operatorname{Tanh}^{m}[x \alpha(t)+\beta(t)] \\
+\sum_{n=0}^{N} F_{n}(t) \cdot \operatorname{Sech}[x \alpha(t)+\beta(t)] \\
\cdot \operatorname{Tanh}^{n}[x \alpha(t)+\beta(t)],
\end{array}
$$

where $E_{m}(t), F_{n}(t), \alpha(t) \neq 0$ and $\beta(t)$ are all differentiable functions. The $x$-linear form and the like are only for the purpose of the easier work.

Since the underlying mechanism for the solitonic features to occur is that different effects that act to change wave forms, i. e., dispersion, dissipation, and nonlinearity, either separately or in various combinations, are able to exactly balance out, we are able to determine the integers $M$ and $N$ via the balance of the highest-order contributions in (1): (i) The highest-order contributions from $u_{x x}$ are among $\operatorname{Tanh}^{M+2} \Psi$ and $\operatorname{Sech} \Psi \operatorname{Tanh}^{N+2} \Psi$; (ii) The highestorder contributions from $u^{3}$ are among $\operatorname{Tanh}^{3 M} \Psi$ and $\operatorname{Sech} \Psi \operatorname{Tanh}^{3 N+2} \Psi$; (iii) Other terms do not contribute so highly. Balancing the contributions mentioned in items (i) and (ii), we get

$$
M=1 \text { and } N=0 .
$$

Therefore we have

$$
\begin{aligned}
u(x, t)= & E_{0}(t)+E_{1}(t) \cdot \operatorname{Tanh}[x \alpha(t)+\beta(t)] \\
& +F_{0}(t) \cdot \operatorname{Sech}[x \alpha(t)+\beta(t)],
\end{aligned}
$$

with at least $E_{1}(t)$ or $F_{0}(t)$ being non-zero, thus giving rise to three possibilities as follows:

Case I - Family I: $F_{0}=0$ but $E_{1} \neq 0$. 
We substitute Ansatz (3) into (1) with computerized symbolic computation, equating to zero the coefficients of like powers of $\operatorname{Tanh} \Psi$ and $x$, so that

$x^{2} \operatorname{Tanh}^{3} \Psi: \alpha^{\prime}(t)=0$ or $\alpha=$ real constant,

where the prime represents derivative with respect to $t$, so that we must have

$$
\beta^{\prime}(t) \neq 0
$$

in order to make the expected solutions meaningful. Next we get

$\operatorname{Tanh}^{3} \Psi: E_{1}(t)=\kappa \sqrt{-2\left[\frac{a \alpha^{2}+\beta^{\prime}(t)^{2}}{d}\right]}, \kappa= \pm 1,(6)$

$\operatorname{Tanh}^{2} \Psi: E_{0}(t)=\frac{1}{6 d\left[a \alpha^{2}+\beta^{\prime}(t)^{2}\right]^{2}}\left[-\sqrt{2} a \alpha^{2} d \kappa \sqrt{\frac{-\left(a \alpha^{2}\right)-\beta^{\prime}(t)^{2}}{d}} \beta^{\prime \prime}(t)-2 a^{2} \alpha^{4} c-4 a \alpha^{2} c \beta^{\prime}(t)^{2}\right.$

$$
\left.-2 c \beta^{\prime}(t)^{4}-3 \sqrt{2} d \kappa \beta^{\prime}(t)^{2} \sqrt{\frac{-\left(a \alpha^{2}\right)-\beta^{\prime}(t)^{2}}{d}} \beta^{\prime \prime}(t)\right] .
$$

Inequality 7 ensures that $E_{1}(t)$ be real. From the coefficients of the terms of $\operatorname{Tanh} \Psi$, we get

$$
\begin{aligned}
& 6 a^{2} \alpha^{4}\left(c^{2}+8 a \alpha^{2} d-3 b d\right) \beta^{\prime}(t)^{2}+2\left(c^{2}+24 a \alpha^{2} d-3 b d\right) \beta^{\prime}(t)^{6}-6 d \beta^{\prime}(t)^{5} \beta^{\prime \prime \prime}(t)-6 a^{2} \alpha^{4} d \beta^{\prime}(t) \beta^{\prime \prime \prime}(t) \\
& +12 d \beta^{\prime}(t)^{8}+a^{2} \alpha^{4}\left[2 a \alpha^{2}\left(c^{2}+6 a \alpha^{2} d-3 b d\right)-5 d \beta^{\prime \prime}(t)^{2}\right]+3 \beta^{\prime}(t)^{4}\left[2 a \alpha^{2}\left(c^{2}+12 a \alpha^{2} d-3 b d\right)+3 d \beta^{\prime \prime}(t)^{2}\right] \\
& \quad-12 a \alpha^{2} d \beta^{\prime}(t)^{3} \beta^{\prime \prime \prime}(t)=0
\end{aligned}
$$

and from the coefficients of the terms of $\operatorname{Tanh}^{0} \Psi$ we see that

$$
\begin{aligned}
& 324 \sqrt{2} d \kappa \beta^{\prime}(t)^{10} \beta^{\prime \prime}(t)-162 \sqrt{2} a^{3} \alpha^{6} d \kappa \beta^{\prime}(t) \beta^{\prime \prime}(t) \beta^{\prime \prime \prime}(t)-162 \sqrt{2} a^{2} \alpha^{4} d \kappa \beta^{\prime}(t)^{3} \beta^{\prime \prime}(t) \beta^{\prime \prime \prime}(t) \\
& +162 \sqrt{2} a \alpha^{2} d \kappa \beta^{\prime}(t)^{5} \beta^{\prime \prime}(t) \beta^{\prime \prime \prime}(t)+162 \sqrt{2} d \kappa \beta^{\prime}(t)^{7} \beta^{\prime \prime}(t) \beta^{\prime \prime \prime}(t) \\
& +2 \beta^{\prime}(t)^{8}\left\{2 c\left(-2 c^{2}+9 b d\right) \sqrt{-\frac{a \alpha^{2}+\beta^{\prime}(t)^{2}}{d}}+9 \sqrt{2}\left(c^{2}+78 a \alpha^{2} d-3 b d\right) \kappa \beta^{\prime \prime}(t)-27 \sqrt{2} d \kappa \beta^{\prime \prime \prime \prime}(t)\right\} \\
& +\beta^{\prime}(t)^{6}\left\{12 \sqrt{2} a \alpha^{2}\left(5 c^{2}+198 a \alpha^{2} d-15 b d\right) \kappa \beta^{\prime \prime}(t)-81 \sqrt{2} d \kappa \beta^{\prime \prime}(t)^{3}\right. \\
& \left.+4 a \alpha^{2}\left[4 c\left(-2 c^{2}+9 b d\right) \sqrt{-\frac{a \alpha^{2}+\beta^{\prime}(t)^{2}}{d}}-45 \sqrt{2} d \kappa \beta^{\prime \prime \prime \prime}(t)\right]\right\} \\
& +a^{2} \alpha^{4} \beta^{\prime}(t)^{2}\left\{36 \sqrt{2} a \alpha^{2}\left(c^{2}+21 a \alpha^{2} d-3 b d\right) \kappa \beta^{\prime \prime}(t)+333 \sqrt{2} d \kappa \beta^{\prime \prime}(t)^{3}\right. \\
& \left.+4 a \alpha^{2}\left[4 c\left(-2 c^{2}+9 b d\right) \sqrt{-\frac{a \alpha^{2}+\beta^{\prime}(t)^{2}}{d}}-27 \sqrt{2} d \kappa \beta^{\prime \prime \prime \prime \prime}(t)\right]\right\} \\
& +3 a \alpha^{2} \beta^{\prime}(t)^{4}\left\{24 \sqrt{2} a \alpha^{2}\left(c^{2}+27 a \alpha^{2} d-3 b d\right) \kappa \beta^{\prime \prime}(t)+99 \sqrt{2} d \kappa \beta^{\prime \prime}(t)^{3}\right.
\end{aligned}
$$




$$
\begin{gathered}
\left.+8 a \alpha^{2}\left[c\left(-2 c^{2}+9 b d\right) \sqrt{-\frac{a \alpha^{2}+\beta^{\prime}(t)^{2}}{d}}-9 \sqrt{2} d \kappa \beta^{\prime \prime \prime \prime}(t)\right]\right\} \\
+a^{3} \alpha^{6}\left\{6 \sqrt{2} a \alpha^{2}\left(c^{2}+18 a \alpha^{2} d-3 b d\right) \kappa \beta^{\prime \prime}(t)-53 \sqrt{2} d \kappa \beta^{\prime \prime}(t)^{3}\right. \\
\left.+2 a \alpha^{2}\left[2 c\left(-2 c^{2}+9 b d\right) \sqrt{-\frac{a \alpha^{2}+\beta^{\prime}(t)^{2}}{d}}-9 \sqrt{2} d \kappa \beta^{\prime \prime \prime \prime}(t)\right]\right\}=0 .
\end{gathered}
$$

This way we have found the first family of exact analytic solutions of (1), which is solitonic, as follows:

$$
\begin{aligned}
u(x, t)= & \kappa \sqrt{-2 \frac{a \alpha^{2}+\beta^{\prime}(t)^{2}}{d}} \operatorname{Tanh}[\alpha x+\beta(t)]-\frac{1}{6 d\left[a \alpha^{2}+\beta^{\prime}(t)^{2}\right]^{2}}\left\{2 c \beta^{\prime}(t)^{4}\right. \\
& \left.+a \alpha^{2}\left[2 a \alpha^{2} c+d \kappa \sqrt{-2 \frac{a \alpha^{2}+\beta^{\prime}(t)^{2}}{d}} \beta^{\prime \prime}(t)\right]+\beta^{\prime}(t)^{2}\left[4 a \alpha^{2} c+3 d \kappa \sqrt{-2 \frac{a \alpha^{2}+\beta^{\prime}(t)^{2}}{d}} \beta^{\prime \prime}(t)\right]\right\}
\end{aligned}
$$

which needs to satisfy 4 constraints, i. e.,

$$
\left\{\begin{array}{l}
\text { Inequality (5), } \\
\text { Inequality (7), } \\
(9), \\
(10) .
\end{array}\right.
$$

In fact, there are 2 classes in this family, for $\kappa= \pm 1$.

\section{Samples of Family I}

Solitary waves can be chosen as the samples to represent this family, with the travelling-wave assumption

$$
\beta(t)=\omega+\gamma t,
$$

where $\gamma \neq 0$ and $\omega$ are real constants. Then,

$$
\text { (10): } c\left(2 c^{2}-9 b d\right)=0 \text {, }
$$

of which 2 cases can be separated:

Family I- $(a): \quad c=0$.

$$
\begin{aligned}
\text { (9): } & 2 a \alpha^{2}-b+2 \gamma^{2}=0 \\
\text { or } \quad & \gamma=\sigma \sqrt{(b / 2)-a \alpha^{2}} \\
& \text { with } \sigma= \pm 1 \\
& b-2 a \alpha^{2}>0 \\
& \text { (to make } \gamma \text { real), }
\end{aligned}
$$$$
\text { Inequality (7): } \quad b / d<0 \text {. }
$$

Hence, we obtain the Family I-(a) solitary-wave solutions of (1),

$$
u(x, t)=\kappa \sqrt{-\frac{b}{d}} \operatorname{Tanh}\left(\omega+\sigma \sqrt{\frac{b}{2}-a \alpha^{2}} t+\alpha x\right)
$$

with the following constraints:

$$
\frac{b}{d}<0, c=0 \text { and } b-2 a \alpha^{2}>0 .
$$

There are 4 classes in Family I-(a), since $\kappa= \pm 1$ and $\sigma= \pm 1$ independently.

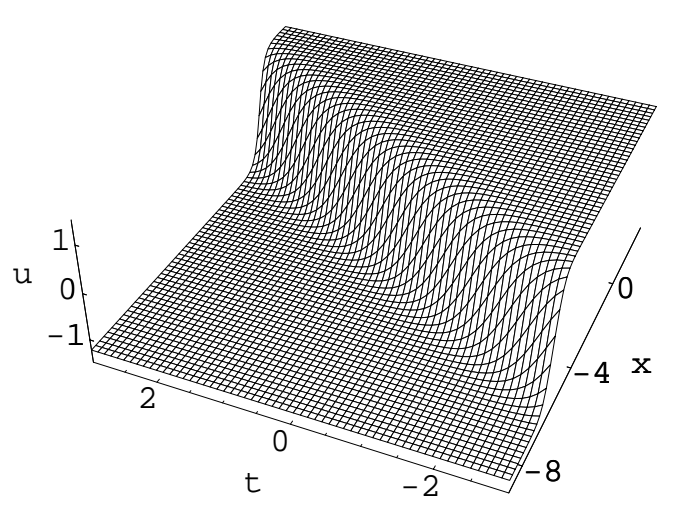

Fig. 1. Observable shock-wave surface $u(x, t)$ for (17) with $\sigma=-1, \kappa=1, b=3, d=-2, a=-1, \alpha=2, c=0$ and $\omega=5$. 


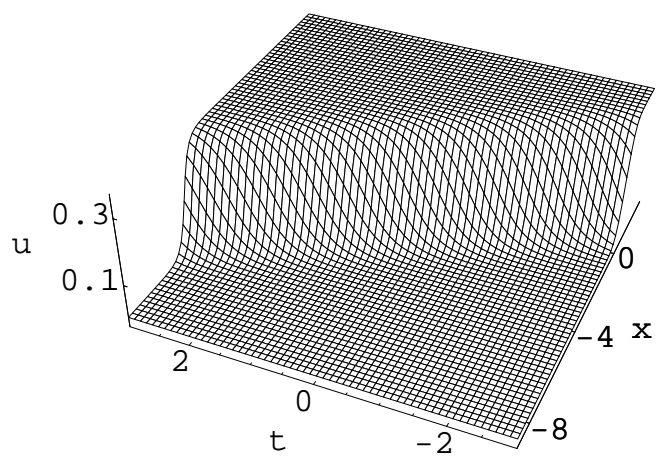

Fig. 2. Observable shock-wave surface $u(x, t)$ for (22), with $\sigma=1, c=1, \kappa=1, d=-2, a=-1, \alpha=2, \omega=5$, and correspondingly, $b=-\frac{1}{9}$.

Family I-(b): $c \neq 0$ and $b=2 c^{2} / 9 d$.

$$
\begin{aligned}
\text { (9): } & c^{2}+18 a \alpha^{2} d+18 d \gamma^{2}=0 \\
\text { or } & \gamma=\sigma \sqrt{-a \alpha^{2}-c^{2} /(18 d)} \\
& \text { with } \sigma= \pm 1 \\
& 18 a \alpha^{2}+c^{2} / d<0 \\
& \text { (to make } \gamma \text { real), }
\end{aligned}
$$

Inequality (7): satisfied already.

We thus get the Family $I-(b)$ solitary-wave solutions of (1)

$$
\begin{array}{r}
u(x, t)=\frac{\kappa}{3} \sqrt{\frac{c^{2}}{d^{2}}} \operatorname{Tanh}\left(\omega+\sigma \sqrt{-a \alpha^{2}-\frac{c^{2}}{18 d}} t\right. \\
+\alpha x)-\frac{c}{3 d}
\end{array}
$$

with the constraints

$$
b=\frac{2 c^{2}}{9 d} \not 0 \text { and } 18 a \alpha^{2}+\frac{c^{2}}{d}<0 .
$$

There are 4 classes in Family I-(b), since $\kappa= \pm 1$ and $\sigma= \pm 1$ independently. Family I-(b) is in line with that reported in [4].

Case II: $F_{0} \neq 0$ but $E_{1}=0$

Into (1) we again substitute Ansatz (3) with symbolic computation, but this time we equate to zero not only the coefficients of like powers of $\operatorname{Tanh} \Psi$ and $x$, but also of like powers of $\operatorname{Sech} \Psi$ :

$x^{2} \operatorname{Sech}^{1} \Psi \operatorname{Tanh}^{2} \Psi: \alpha^{\prime}(t)=0$ or $\alpha=$ real

constant, so that $\beta^{\prime}(t) \neq 0$,

$\operatorname{Sech}^{0} \Psi \operatorname{Tanh}^{2} \Psi: E_{0}=-\frac{c}{3 d}$,

$\operatorname{Sech}^{0} \Psi \operatorname{Tanh}^{0} \Psi: c\left(2 c^{2}-9 b d\right)=0$.

In fact (25) leads to two families as follows:

Family II- $(a): c=0$.

Continue the work, and we get

$\operatorname{Sech}^{1} \Psi \operatorname{Tanh}^{2} \Psi: F_{0}=\kappa \sqrt{2 \frac{a \alpha^{2}+\beta^{\prime}(t)^{2}}{d}}$

$$
\text { with } \kappa= \pm 1 \text { and } \frac{a \alpha^{2}+\beta^{\prime}(t)^{2}}{d}>0 \text {. }
$$

$\operatorname{Sech}^{1} \Psi \operatorname{Tanh}^{1} \Psi:\left[a \alpha^{2}+3 \beta^{\prime}(t)^{2}\right] \beta^{\prime \prime}(t)=0$

$$
\Rightarrow \beta=\omega+\gamma t \text {, where } \gamma \neq 0 \text { and }
$$

$\omega$ are real constants.

$\operatorname{Sech}^{1} \Psi \operatorname{Tanh}^{0} \Psi: \gamma=\sigma \sqrt{-a \alpha^{2}-b}$

$$
\text { with } \sigma= \pm 1 \text { and } a \alpha^{2}+b<0 \text {. }
$$

We report the Family II-(a) solitary-wave solutions of (1),

$$
u(x, t)=\kappa \sqrt{-\frac{2 b}{d}} \operatorname{Sech}\left(\omega+\sigma \sqrt{-a \alpha^{2}-b} t+\alpha x\right)
$$

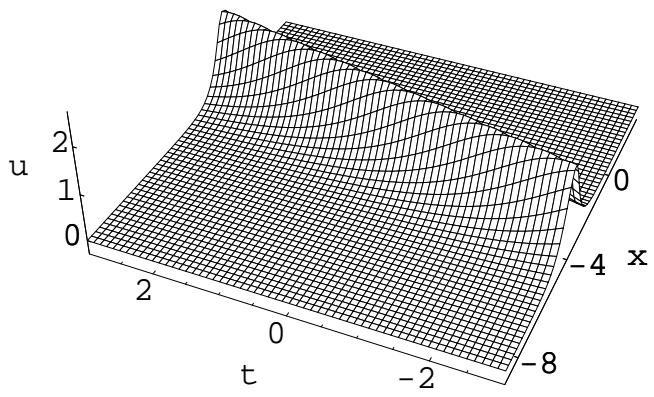

Fig. 3. Observable bell-shaped solitary-wave surface $u(x, t)$ for (29). The values of the parameters are the same as those in Fig. 1. 


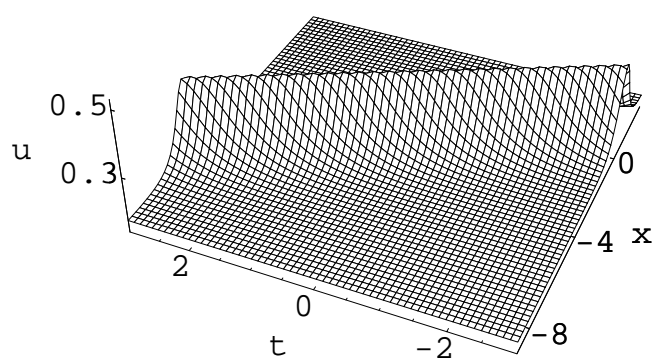

Fig. 4. Observable bell-shaped solitary-wave surface $u(x, t)$ for (33). The values of the parameters are the same as those in Fig. 2.

with the following constraints:

$$
\frac{b}{d}<0, c=0 \text { and } a \alpha^{2}+b<0
$$

There are 4 classes in this family, since $\kappa= \pm 1$ and $\sigma= \pm 1$, respectively.

Family II-(b): $c \neq 0$ and $b=2 c^{2} / 9 d$.

This time we see that

$\operatorname{Sech}^{1} \Psi \operatorname{Tanh}^{2} \Psi: F_{0}=\kappa \sqrt{2 \frac{a \alpha^{2}+\beta^{\prime}(t)^{2}}{d}}$

$$
\text { with } \kappa= \pm 1 \text { and } \frac{a \alpha^{2}+\beta^{\prime}(t)^{2}}{d}>0 \text {. }
$$

$\operatorname{Sech}^{1} \Psi \operatorname{Tanh}^{1} \Psi: \beta=\omega+\gamma t$, where $\gamma \neq 0$ and $\omega$ are real constants.

$\operatorname{Sech}^{1} \Psi \operatorname{Tanh}^{0} \Psi: \gamma=\sigma \sqrt{\frac{c^{2}}{9 d}-a \alpha^{2}}$

$$
\text { with } \sigma= \pm 1 \text { and } \frac{c^{2}}{9 d}-a \alpha^{2}>0 \text {. }
$$

Therefore we end up with the Family II-(a) solitarywave solutions of (1):

$$
u(x, t)=\frac{\kappa}{3} \sqrt{\frac{2 c^{2}}{d^{2}}} \operatorname{Sech}\left(\omega+\sigma \sqrt{\frac{c^{2}}{9 d}-a \alpha^{2}} t\right.
$$

with the constraints:

$$
+\alpha x)-\frac{c}{3 d}
$$

$$
b=\frac{2 c^{2}}{9 d} \neq 0, \text { and } \frac{c^{2}}{9 d}-a \alpha^{2}>0 .
$$

In fact, there are 4 classes in this family, since $\kappa= \pm 1$ and $\sigma= \pm 1$ respectively. Family II-(b) is in line with that reported in [4].

Case III: $F_{0} \neq 0$ and $E_{1} \neq 0$

It will be shown that in this case we have to consider (1) in the complex field. Substituting Ansatz (3) into (1) with symbolic computation, and we firstly meet

$$
x^{2} \operatorname{Sech}^{1} \Psi \operatorname{Tanh}^{2} \Psi:
$$

$$
\alpha^{\prime}(t)=0 \text { or } \alpha=\text { real constant. }
$$

However, further steps are so complicated that we restrict ourselves to the simplest case (i.e., solitary waves), with

$$
\beta=\omega+\gamma t,
$$

where $\gamma \neq 0$ and $\omega$ are real constants. The reason to make both $\alpha$ and $\beta$ be real is to retain the solitonic profile even with some complex coefficients involved. Then

$\operatorname{Sech}^{1} \Psi \operatorname{Tanh}^{2} \Psi: \quad F_{0}=\kappa \sqrt{\frac{2 a \alpha^{2}+2 \gamma^{2}+3 d E_{1}^{2}}{d}}$ with $\kappa= \pm 1$ and $2 a \alpha^{2}+2 \gamma^{2}+3 d E_{1}^{2} \neq 0$.

$\operatorname{Sech}^{1} \Psi \operatorname{Tanh}^{1} \Psi$ and $\operatorname{Sech}^{0} \Psi \operatorname{Tanh}^{2} \Psi$ :

$$
\begin{aligned}
& E_{0}=-\frac{c}{3 d}, \\
& E_{1}=\text { constant } .
\end{aligned}
$$

$\operatorname{Sech}^{1} \Psi \operatorname{Tanh}^{0} \Psi: E_{1}=\frac{\sigma}{3} \sqrt{\frac{c^{2}-3 d\left(a \alpha^{2}+b+\gamma^{2}\right)}{d^{2}}}$

$$
\text { with } \sigma= \pm 1 \text { and } c^{2} \neq 3 d\left(a \alpha^{2}+b+\gamma^{2}\right) \text {. }
$$

$\operatorname{Sech}^{0} \Psi \operatorname{Tanh}^{0} \Psi: c\left(2 c^{2}-9 b d\right)=0$.

Hereby (39) again leads to two families as follows:

Family III-(a): $c=0$.

Both, the coefficients of $\operatorname{Sech}^{0} \Psi \operatorname{Tanh}^{3} \Psi$ and of $\operatorname{Sech}^{0} \Psi \operatorname{Tanh}^{1} \Psi$ yield

$\gamma=\epsilon \sqrt{2 b-a \alpha^{2}}$ with $\epsilon= \pm 1$ and $2 b>a \alpha^{2}$.

and the Family III-(a) of exact solutions of (1) come out as

$$
\begin{aligned}
u(x, t)= & \kappa \sqrt{\frac{b}{d}} \operatorname{Sech}\left(\omega+\epsilon \sqrt{2 b-a \alpha^{2}} t+\alpha x\right) \\
& +i \sigma \sqrt{\frac{b}{d}} \operatorname{Tanh}\left(\omega+\epsilon \sqrt{2 b-a \alpha^{2}} t+\alpha x\right)
\end{aligned}
$$


with the constraints

$c=0$ and $2 b>a \alpha^{2}$.

This family behaves like solitary waves but possesses a complex coefficient. There are 8 classes in this family, since $\kappa= \pm 1, \epsilon= \pm 1$ and $\sigma= \pm 1$, respectively.

Family III-(b): $c \neq 0$ and $b=2 c^{2} / 9 d$.

Now both the coefficients of $\operatorname{Sech}^{0} \Psi \operatorname{Tanh}^{3} \Psi$ and of $\operatorname{Sech}^{0} \Psi \operatorname{Tanh}^{1} \Psi$ give rise to

$$
\begin{aligned}
& \gamma=\epsilon \sqrt{-a \alpha^{2}-\frac{2 c^{2}}{9 d}} \quad \text { with } \epsilon= \pm 1 \\
& \quad \text { and } a \alpha^{2}+\frac{2 c^{2}}{9 d}<0,
\end{aligned}
$$

so that we figure out the Family III-(b) exact solutions of (1) as

$$
\begin{aligned}
u(x, t)= & \frac{i \kappa}{3} \sqrt{\frac{c^{2}}{d^{2}}} \operatorname{Sech}\left(\omega+\epsilon \sqrt{-a \alpha^{2}-\frac{2 c^{2}}{9 d}} t+\alpha x\right) \\
& +\frac{\sigma}{3} \sqrt{\frac{c^{2}}{d^{2}}} \operatorname{Tanh}\left(\omega+\epsilon \sqrt{-a \alpha^{2}-\frac{2 c^{2}}{9 d}} t+\alpha x\right) \\
& -\frac{c}{3 d}
\end{aligned}
$$

with the constraints:

$$
b=\frac{2 c^{2}}{9 d} \neq 0 \text { and } a \alpha^{2}+\frac{2 c^{2}}{9 d}<0 .
$$

This family also behaves like solitary waves, but possess a complex coefficient. Again, there are 8 classes

[1] D. Zwillinger, Handbook of Differential Equations, Academic Press, London 1989.

[2] C. Gu et al., Soliton Theory and Its Application, Zhejiang Sci. Tech. Press, Hangzhou 1990.

[3] M. Ablowitz et al., Solitons, Nonlinear Evolution Equations and Inverse Scattering, Cambridge Univ. Press, Cambridge 1991. in this family, since $\kappa= \pm 1, \epsilon= \pm 1$ and $\sigma= \pm 1$ respectively. Family III-(b) is in line with that reported in [4].

\section{Conclusions and Discussions}

By virtue of the generalized hyperbolic-function method and computerized symbolic computation, we have obtained certain exact analytic solutions of (1), which are all solitonic.

Some of the solitonic features have been pictured out: Figs. 1 and 2 demonstrate the shock-wave structures for (17) and (22), respectively, while Figs. 3 and 4 illustrate the bell-shaped solitary waves in the sense of (29) and (33), respectively. In all these figures, the numerical values chosen for the free parameters are purely for the picture drawing or qualitative analysis.

In reality, the detailed application of the solitonic solutions requires a judicious choice of those parameters, depending on each underlying physics mechanism under investigation.

Finally, it is of special interest to note that the aforementioned solitonic features are observable with the corresponding experiments.

\section{Acknowledgements}

We thank Prof. Q. S. Lu for his valuable help. This work has been supported by the China Talent Fund, by the Cheung Kong Scholars Programme of China, by the Cheung-Kong-Scholars Research Concerted Fund of Beijing University of Aeronautics and Astronautics, and by the Doctoral Education Fund in Basic Sciences of Beijing University of Aeronautics and Astronautics.

[4] Z. Yan and H. Zhang, Comm. Non. Sci. and Num. Sim. 4, 224 (1999).

[5] C. Hua and Q. S. Lu, Appl. Math. and Mech. 21, 925 (2000).

[6] Y. T. Gao and B. Tian, Computer Phys. Comm. 133, 158 (2001); Int. J. Mod. Phys. C 12, 891 (2001); Y. T.. Gao, B. Tian, and G. W. Wei, Int. J. Mod. Phys. C 12, 1417 (2001). 